\title{
Dendroécologie du pin laricio de Corse dans l'ouest de la France. Évolution du potentiel de croissance au cours des dernières décennies
}

\author{
F Lebourgeois, M Becker
}

Unité d'écophysiologie forestière, équipe phytoécologie, Inra-Nancy, 54280 Champenoux, France

(Reçu le 19 mai 1995 ; accepté le 7 septembre 1995)

\begin{abstract}
Summary - Dendroecological study of Corsican pine in western France. Growth potential evolution during the last decades. A total of 1808 Corsican pines (Pinus nigra Arnold ssp laricio Poiret var corsicana) were sampled in western France (Pays-de-la Loire region). The evolution of the mean growth of each tree and the relationship between the radial growth and the present crown conditions were studied. For each ring, earlywood and latewood widths were measured separately. The analysis of the mean radial growth in relation to the cambial age showed that the young rings ( $\leq 10$ years) were mainly made up of earlywood ( 70 to $80 \%$ ). It was only from 40 years onwards that the percentages of each ring compartment become equal $(50 \%)$. The radial growth mean curves from 1935 to 1991 showed i) strong annual variations, in relation to annual climatic conditions; ii) several crises of varying severity and length, on the decade time scale, during which growth declined because of sustained climatic stresses $(1952,1962$ and since 1981); iii) an increasing general trend of the growth indices $+50,+60$ and $+35 \%$ for, respectively, the total ring, the earlywood and the latewood. These results confirm the long-term growth trends observed in several French regions for various species, which may be interpreted as a result of global climatic changes (as a consequence of the increase in atmospheric $\mathrm{CO}_{2}$, atmospheric anthropogenic deposits, especially of nitrogen compounds, and improvement of silviculture (selection of seedlings, thinning, density of plantation). By stratification of the whole sample according to crown condition, divergent curves were obtained. The loss of vitality of the now declining trees occurred from the end of the 1960 s onwards in relation to the succession of years characterized by a pronounced rainfall deficit (1967-1978). The impact of these dry years on the declining trees has been particularly amplified by locally unfavourable water site conditions. The importance of crown condition in assessing the current vigour of trees is also discussed.
\end{abstract}

Pinus nigra / dendrochronology / radial growth / earlywood / latewood / growth trend / decline l climate

Résumé - L'évolution de la croissance radiale moyenne de 1808 pins laricio de Corse (Pinus nigra Arnold ssp laricio Poiret var corsicana) échantillonnés dans 183 placettes de l'ouest de la France (région Pays-de-la-Loire) a été étudiée, ainsi que les relations entre la croissance et l'état actuel des houppiers. Pour chaque cerne, la largeur du bois initial et celle du bois final ont été mesurées séparément. L'accroissement annuel moyen selon l'âge cambial montre que les jeunes cernes ( 10 ans) sont constitués principalement de bois initial ( 70 à $80 \%$ ). Ce n'est qu'à partir de 40 ans que les pourcentages des deux compartiments dans chaque cerne s'égalisent (50\%). Entre 1935 et 1991 , 
les courbes moyennes de croissance radiale montrent $;$ i) de fortes variations interannuelles en liaison avec les conditions climatiques; ii) des crises variables en durée et en intensité, liées à des stress climatiques prolongés $(1952,1962$, depuis 1981); iii) une tendance générale positive respectivement de $+50,+60$ et $+35 \%$ si l'on considère le cerne total, le bois initial et le bois final. Ces résultats confirment les tendances déjà observées sur d'autres espèces dans d'autres régions françaises, et peuvent être interprétés comme les conséquences des modifications climatiques globales plus ou moins directement liées à l'augmentation du $\mathrm{CO}_{2}$ atmosphérique, des dépôts atmosphériques anthropiques, particulièrement azotés, ou encore de lamélioration de la sylviculture (sélection des plants, densité de plantation, éclaircies...). La stratification des données selon l'aspect actuel des houppiers révèle une divergence de croissance depuis la fin des années 1960 des peuplements actuellement déclinants. Cette perte ancienne de vitalité semble être en relation étroite avec la succession des déficits pluviométriques des années 1967 à 1978 ; déficits dont les effets ont été amplifiés pour ces peuplements par des conditions locales d'approvisionnement en eau défavorables. La pertinence du critère aspect des houppiers pour juger de la vitalité réelle des arbres est discutée.

Pinus nigra / dendrochronologie / croissance radiale / bois initial / bois final / changement de croissance / dépérissement / climat

\section{INTRODUCTION ET OBJECTIFS}

Les symptômes de " dépérissement des forêts " observés au début des années 1970 sur le sapin et l'épicéa en Allemagne, puis à partir de 1983 dans les Vosges, ont suscité depuis plus de 10 ans de très nombreux travaux de recherches dans la communauté scientifique internationale. En France, c'est dans le cadre du programme Deforpa (Dépérissement des forêts attribué à la pollution atmosphérique) (Bonneau, 1987) que les premières études reposant sur l'analyse rétrospective de la croissance radiale des peuplements (études dendroécologiques) ont été menées, sur le sapin dans les Vosges (Becker, 1987, 1989) et dans le Jura (Bert et Becker, 1990 ; Bert, 1992).

Sans disculper pour autant la pollution atmosphérique, ces travaux ont surtout mis en évidence l'importance du climat (sécheresses principalement) et de la sylviculture dans les pertes de vitalité et les symptômes de dépérissement observés. Au-delà de ces « accidents » observés à l'échelle de l'année ou de la décennie, ils ont aussi conclu, de façon plus inattendue, à l'existence d'une forte dérive positive à long terme, à l'échelle du siècle, de la croissance radiale annuelle. Afin d'éprouver la portée de ces résultats, d'autres études ont été entreprises impliquant d'autres espèces et/ou des contextes climatiques et édaphiques différents : hêtre dans les Vosges (Picard, 1995), chêne (Becker et al, 1994) et hêtre (Badeau et al, 1995) sur le plateau lorrain. Toutes confirment l'existence de la ten-dance à long terme décelée précédemment. La productivité ligneuse des écosystèmes forestiers naturels du nord-est de la France, résineux et feuillus, semble bien avoir fortement augmenté depuis une centaine d'années. Selon les cas, la crois-sance radiale actuelle est en moyenne de $55 \%$ à $160 \%$ supérieure à celle observée à la fin du siècle dernier. Des résultats comparables ont également été obtenus en Europe et en Amérique (Hari et al, 1984 ; Hari et Arovaara, 1988 ; Graumlich et al, 1989 ; Graumlich, 1991 ; Briffa, 1992 ; Kauppi et al, 1992).

Il est apparu utile de savoir si, en France, un tel phénomène s'observait aussi dans des contextes climatiques plus radicalement différents et/ou chez des espèces " non naturelles " largement utilisées en reboisement. La réponse à cette question constitue l'un des objectifs de l'étude présentée ici.

II s'agit là aussi d'une étude dendroécologique, c'est-à-dire associant étroitement 
la dendrochronologie (fondée sur la mesure des cernes d'accroissement annuels) et la phytoécologie (analyse des conditions physiques et biotiques de croissance). L'espèce choisie est le pin laricio de Corse (Pinus nigra Arnold ssp laricio Poiret var corsicana) et le contexte bioclimatique étudié est celui de la région Pays-de-la-Loire, dans l'ouest de la France. Un autre objectif important de l'étude est en effet d'approfondir les connaissances sur l'autécologie de cette importante essence de reboisement, qui occupe actuellement la troisième place au niveau national et la première dans la région d'étude (Durel et Charon, 1994). Les aptitudes connues du pin laricio, liées à ses origines bioclimatiques (montagnes méditerranéennes), permettent de penser que ce choix peut être judicieux dans de nombreuses régions de l'ouest et du Centre de la France. Il est par ailleurs probable que son utilisation prenne une importance grandissante dans le contexte actuel de la déprise de nombreuses terres agricoles.

Enfin, bien qu'à ce jour aucun symptôme alarmant de dépérissement n'ait été signalé sur le pin laricio de Corse dans la région Pays-de-la-Loire, nous avons pris en compte dans l'étude l'état sanitaire des peuplements échantillonnés. Récemment, des mortalités importantes ont en effet affecté d'autres espèces, en particulier le douglas (Pseudotsuga menziesii [Mirb] Franco), probablement à la suite des sécheresses prolongées de 1989 à 1991. Si les scénarios les plus couramment avancés aujourd'hui au sujet des changements climatiques globaux viennent à se confirmer, les pins, et le pin laricio de Corse en particulier, pourraient se révéler des essences de substitution de premier plan dans une gamme encore élargie de milieux.

Ce premier article présente les résultats relatifs à l'évolution moyenne de la croissance radiale du pin laricio de Corse dans la région Pays-de-la-Loire depuis une soixantaine d'années, ainsi que les relations entre l'aspect actuel des houppiers et la croissance radiale.

\section{AIRE D'ÉTUDE ET ÉCHANTILLONNAGE}

L'étude porte sur 183 placettes réparties dans trois des cinq départements de la région Pays-de-la-Loire (fig 1). Les placettes, installées dans des plantations pures de pin laricio de Corse, ont été choisies de façon à couvrir toute la diversité des situations écologiques possibles (altitude, exposition, pente, position topographique, classe de drainage, fertilité minérale des sols et types stationnels...). À partir de la description détaillée du sol (épaisseur, texture et structure des horizons, pourcentage de cailloux...), et de l'observation du système racinaire sur le profil (profondeur d'enracinement et densité racinaire pour chaque horizon décrit), un indice de réserve utile en eau a été calculé sur chaque placette (Lebourgeois, 1995).

Sur chaque placette, dix arbres dominants bien conformés ont été sélectionnés. La hauteur et la circonférence de chaque arbre ont été mesurées, et un carottage à la moelle à $1,30 \mathrm{~m}$ du sol a été effectué à l'aide d'une tarière de Pressler. Au total, 1808 pins ont été échantillonnés sur les 183 placettes. Le pin laricio de Corse n'ayant été massivement utilisé dans les reboisements qu'à partir des années 1950, il n'existe pas de très vieux peuplements dans la région d'étude. Les plus anciennes plantations qui ont pu être échantillonnées ont au plus 70 ans (à $1,30 \mathrm{~m}$ ), et l'âge moyen des 1808 pins étudiés est de 30 ans (fig 2).

Compte tenu de la part importante de subjectivité inhérente à toute estimation de l'état sanitaire des arbres et particulièrement du degré de défoliation, et du fait que plusieurs notateurs ont été impliqués dans l'étude, nous avons préféré attribuer une note sanitaire globale à la placette, plutôt 


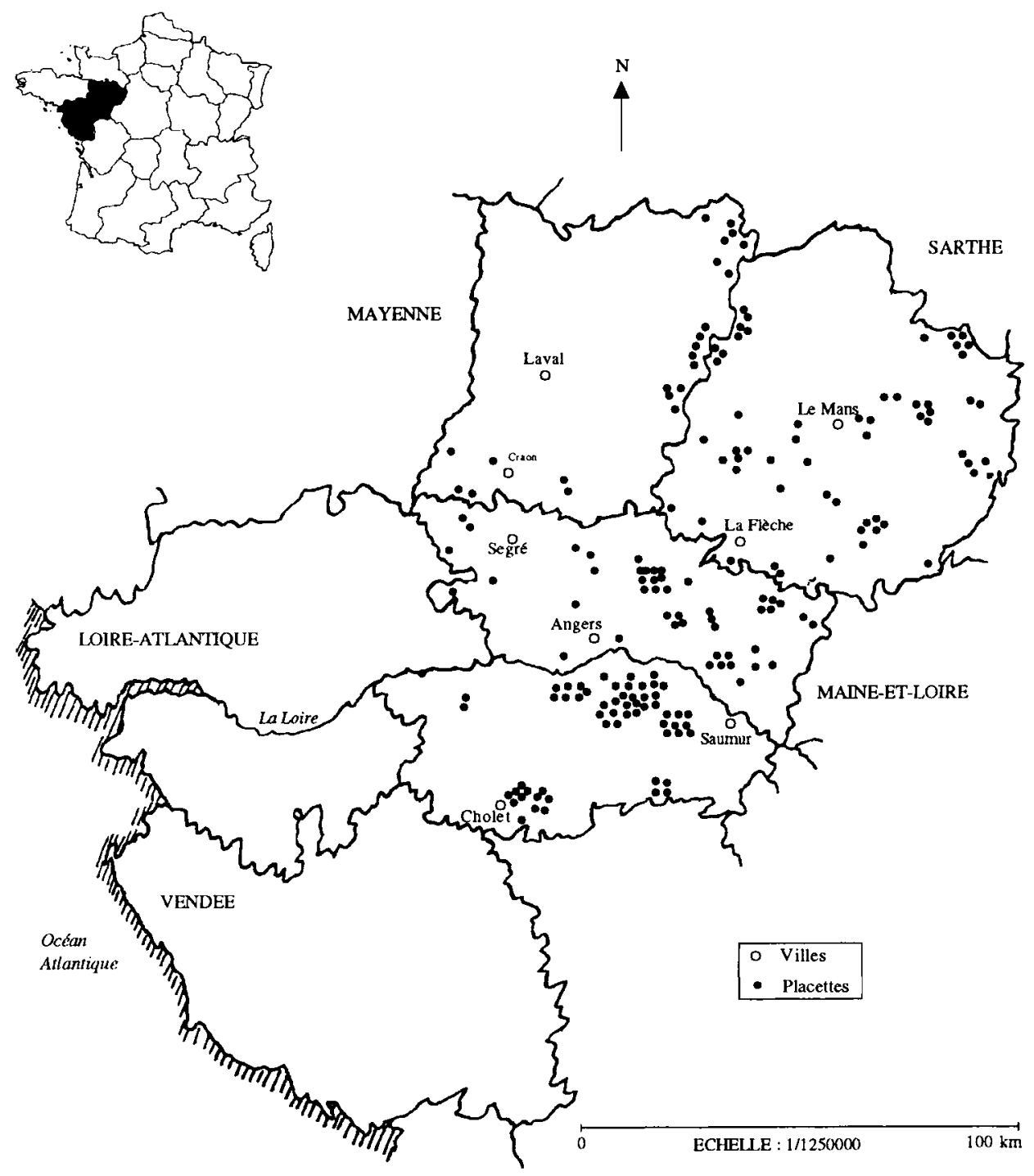

Fig 1. Localisation géographique des 183 placettes.

que de noter individuellement chaque arbre. L'état sanitaire correspond ici au pourcentage d'arbres de la placette présentant un pourcentage de pertes foliaires compris entre 5 et $25 \%$. Le chiffre maximum de $25 \%$ d'aiguilles manquantes (par rapport à la quantité que l'arbre devrait avoir s'il était parfaitement sain) a été déterminé a pos- 


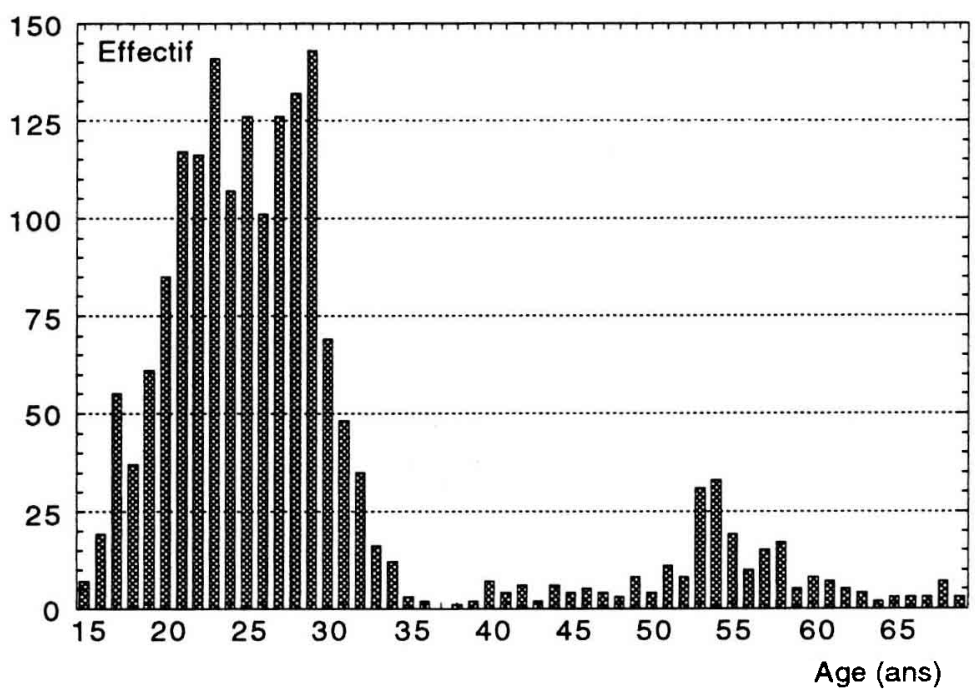

Fig 2. Répartition de l'effectif des arbres selon l'âge (à 1,30 m).

teriori, et correspond à la limite supérieure de pertes foliaires observées sur le terrain. L'échelle de notation de l'état sanitaire et l'effectif de placettes pour chaque classe sont présentés dans le tableau 1.

\section{MÉTHODES}

Avant la mesure des cernes, les 1808 carottes ont été "planées ". Le planage se fait après réhumidification de la carotte, et dans le plan perpendiculaire au fil du bois. Cette opération consiste à éliminer à l'aide d'une lame tranchante environ un tiers de l'épaisseur des é- chantillons. Un planage soigné assure une parfaite observation des moindres détails de la structure du bois, et permet de mieux révéler d'éventuels cernes très fins.

Un total de 49379 cernes ont été mesurés à l'aide d'un système vidéo-informatisé (précision théorique : $1 / 100^{\mathrm{e}} \mathrm{mm}$ ). Nous avons mesuré séparément, pour chaque cerne, la largeur du bois initial (zone claire du cerne) et la largeur du bois final (zone foncée du cerne) ; la largeur totale étant la somme de ces deux largeurs. Ceci devait permettre de mettre en évidence d'éventuelles différences de comportement des deux composantes du cerne vis-à-vis des conditions climatiques régionales, mais également, dans le

Tableau I. Échelle de notation de l'état sanitaire et effectif des placettes pour chaque classe. Les défoliations observées sont comprises entre 5 et $25 \%$.

\begin{tabular}{llc}
\hline Modalité & & Nombre de placettes \\
\hline Aucune perte foliaire & Classe 1 & 113 \\
1 ou 2 arbres défoliés & Classe 2 & 50 \\
3 ou 4 arbres défoliés & Classe 3 & 14 \\
Plus de 5 arbres défoliés & Classe 4 & 1 \\
Tous les arbres défoliés & Classe 5 & 5 \\
\hline
\end{tabular}


cas où une évolution à long terme de la croissance serait décelée, de voir si celle-ci s'exprime de la même façon quel que soit le compartiment du cerne considéré.

Après la mesure, les profils dendrochronologiques sont interdatés (= synchronisés) afin de vérifier que chaque cerne correspond bien à la date réelle de son élaboration (Fritts, 1976 ; Becker, 1987). Le principe de cette interdatation repose sur l'utilisation d'années caractéristiques pour lesquelles la grande majorité des arbres présente le même comportement (Schweingruber, 1985, 1992 ; Schweingruber et al, 1989). Pour notre étude, une année $n$ a été considérée comme « caractéristique » quand la largeur du cerne $n$ était différente d'au moins $10 \%$ (en plus ou en moins) de celle du cerne formé l'année $n-1$, et qu'au moins $65 \%$ des arbres présentaient le même comportement pour cette année. Elle a été définie comme « très caractéristique " quand au moins $75 \%$ des arbres réagissaient de la même façon.

À partir de la largeur de chaque cerne et de sa distance au cœur de l'arbre, on calcule ensuite la surface de l'anneau correspondant (en $\mathrm{cm}^{2}$ ). Les surfaces de cernes donnent en effet une meilleure estimation de l'accroissement en biomasse que les largeurs (Pardé et Bouchon, 1988 ; Briffa, 1992 ; Becker et al, 1994). Elles permettent de corriger l'effet de la décroissance géométrique de la largeur en fonction de la position relative du cerne par rapport au centre. Ceci ne permet cependant pas d'éliminer totalement l'effet du vieillissement de l'arbre. Pour pouvoir comparer valablement les surfaces de cernes, il est indispensable d'éliminer la part de variation qui demeure liée à l'âge cambial (âge de l'arbre au moment où le cerne a été élaboré) (Fritts, 1976). La prise en compte de cet effet âge implique la mise en œuvre d'une méthode de standardisation.

La méthode de standardisation utilisée ici est celle qui a fait ses preuves depuis quelques années dans l'analyse des signaux de basse fréquence véhiculés par les séries chronologiques (Becker, 1989 ; Bert, 1992 ; Dupouey et al, 1992 ; Becker et al, 1994 ; Badeau, 1995 ; Picard, 1995). Elle s'opère en deux étapes.

La première étape consiste à calculer, pour chaque âge cambial, la surface moyenne des cernes disponibles sur l'ensemble de l'échantilIon régional. Étant donné la large gamme d'âge actuel des arbres échantillonnés et la variété des milieux rencontrés, à chaque âge cambial correspondent des cernes élaborés par des arbres différents, à des dates très variées, dans des conditions individuelles, stationnelles et climatiques très différentes, qui tendent à se compenser mutuellement. La courbe “âge » moyenne ainsi obtenue exprime donc essentiellement la loi biologique régionale liant la croissance radiale au vieillissement de l'arbre. Dans le processus de calcul, il est souhaitable de tenir compte des effectifs différents de cernes disponibles par date pour chaque âge cambial, afin de donner le même poids à chaque date dans le calcul de la moyenne. Le coefficient de pondération appliqué à chaque cerne lors de la construction de la courbe âge est le rapport de l'effectif moyen de cernes disponibles à la date a (pour les diverses dates où des cernes sont disponibles) et de l'effectif de cernes d'âge a à la date $d$. Un ajustement mathématique de la courbe âge permet ensuite d'éliminer les variations non biologiques aléatoires, ou relatives au faible effectif de cernes disponibles pour les âges cambiaux élevés.

La seconde étape de la standardisation consiste à convertir les surfaces de cernes en indices de croissance radiale dégagés de l'influence de l'âge : chaque indice $I_{c}$ (exprimé en $\%$ ) est le rapport de la surface du cerne mesuré sur la surface moyenne ajustée au même âge cambial, fournie par la courbe âge précédente.

Ensuite, diverses courbes peuvent être calculées en faisant des moyennes d'indices de croissance par date : une courbe de croissance radiale moyenne de l'ensemble des arbres de l'échantillon (courbe de référence), et un ensemble de courbes moyennes partielles, obtenues en stratifiant l'échantillon total selon la valeur prise par divers paramètres mesurés sur le terrain (état sanitaire en particulier dans le cas des résultats présentés ici).

\section{RÉSULTATS}

Les principales années caractéristiques ont été surtout des années à faible croissance $(n=8: 1937,1952,1959,1962$, $1969,1976,1986,1989)$, plus rarement des années à forte croissance $(n=3$ : $1935,1977,1988$ ) (tableau II). La synchronisation des séries chronologiques n'a pas posé de problèmes majeurs. Sur les 1808 carottes mesurées, seules 21 ont dû être 
Tableau II. Années caractéristiques pour le pin laricio de Corse dans la région Pays-de-la-Loire.

\begin{tabular}{lccccc}
\hline \multirow{2}{*}{ Date } & \multicolumn{2}{c}{ Fréquence (\%) de l'évolution interannuelle } & \multirow{2}{*}{$\begin{array}{c}\text { Écart relatif } \\
\text { moyen (\%) }\end{array}$} & $\begin{array}{c}\text { Nombres } \\
\text { d'arbres }\end{array}$ \\
\cline { 2 - 4 } & Augmentation & Diminution & Maintien & & \\
\hline 1989 & 2 & 92 & 4 & -36 & 1808 \\
1988 & 71 & 10 & 18 & 40 & 1808 \\
1986 & 11 & 68 & 20 & -17 & 1808 \\
1977 & 84 & 2 & 12 & 52 & 1773 \\
1976 & 1 & 92 & 5 & -34 & 1718 \\
1969 & 9 & 67 & 22 & -16 & 1012 \\
1962 & 10 & 69 & 19 & -16 & 302 \\
1959 & 1 & 86 & 11 & -29 & 254 \\
1952 & 3 & 87 & 8 & -31 & 233 \\
1937 & 9 & 75 & 15 & -15 & 64 \\
1935 & 78 & 4 & 16 & 36 & 51 \\
\hline
\end{tabular}

corrigées, parmi lesquelles quatre présentaient de vrais cernes manquants (absence d'une à trois cernes sur la section traversée par la carotte).

\section{Accroissement annuel moyen en fonction de l'âge cambial}

\section{Relation surface du cerne complet-âge cambial}

Le nombre important de cernes disponibles et la diversité des classes d'âge des arbres échantillonnés expliquent que la courbe obtenue par simple calcul de la moyenne, pondérée comme il a été précisé plus haut, soit très régulière (fig 3 ). Pendant les 10 premières années, la croissance radiale augmente très rapidement et passe de 0,7 à $11,6 \mathrm{~cm}^{2} / \mathrm{an}$. De 10 à 35 ans, l'augmentation est beaucoup moins importante mais reste régulière, et la surface du cerne moyen atteint $21 \mathrm{~cm}^{2}$ à 35 ans. De 35 à 60 ans, l'augmentation fléchit légèrement et les variations deviennent plus importantes, en raison principalement du plus faible effectif de cernes disponibles; avec la surface du cerne moyen, elle est de l'ordre de $25 \mathrm{~cm}^{2}$ à 60 ans.
Après 60 ans, la courbe présente une chute brutale et très importante. Ce phénomène ne correspond cependant pas à une réalité biologique normale caractérisant l'effet de l'âge cambial. II est la conséquence, d'une part, du faible effectif de cernes disponible pour ces âges ( 3 à 25 cernes), d'autre part, et surtout, du fait que ces cernes de plus de 60 ans d'âge cambial correspondent à des arbres appartenant à une unique classe d'âge ( 60 à 70 ans) et que la plupart ont été élaborés entre 1985 et 1991 ; période caractérisée par des conditions climatiques très défavorables pour la croissance (hivers froids ; sécheresses printanières et estivales). Au-delà de 60 ans, la courbe n'étant plus statistiquement fiable, c'est donc l'ajustement précédent qui a été prolongé pour le calcul des indices de croissance.

\section{Relation surface des cernes-âge cambial pour le bois initial et le bois final}

Pour le bois initial, l'allure générale de la courbe traduisant l'évolution de l'accroissement annuel moyen selon l'âge cambial est très proche de celle observée 


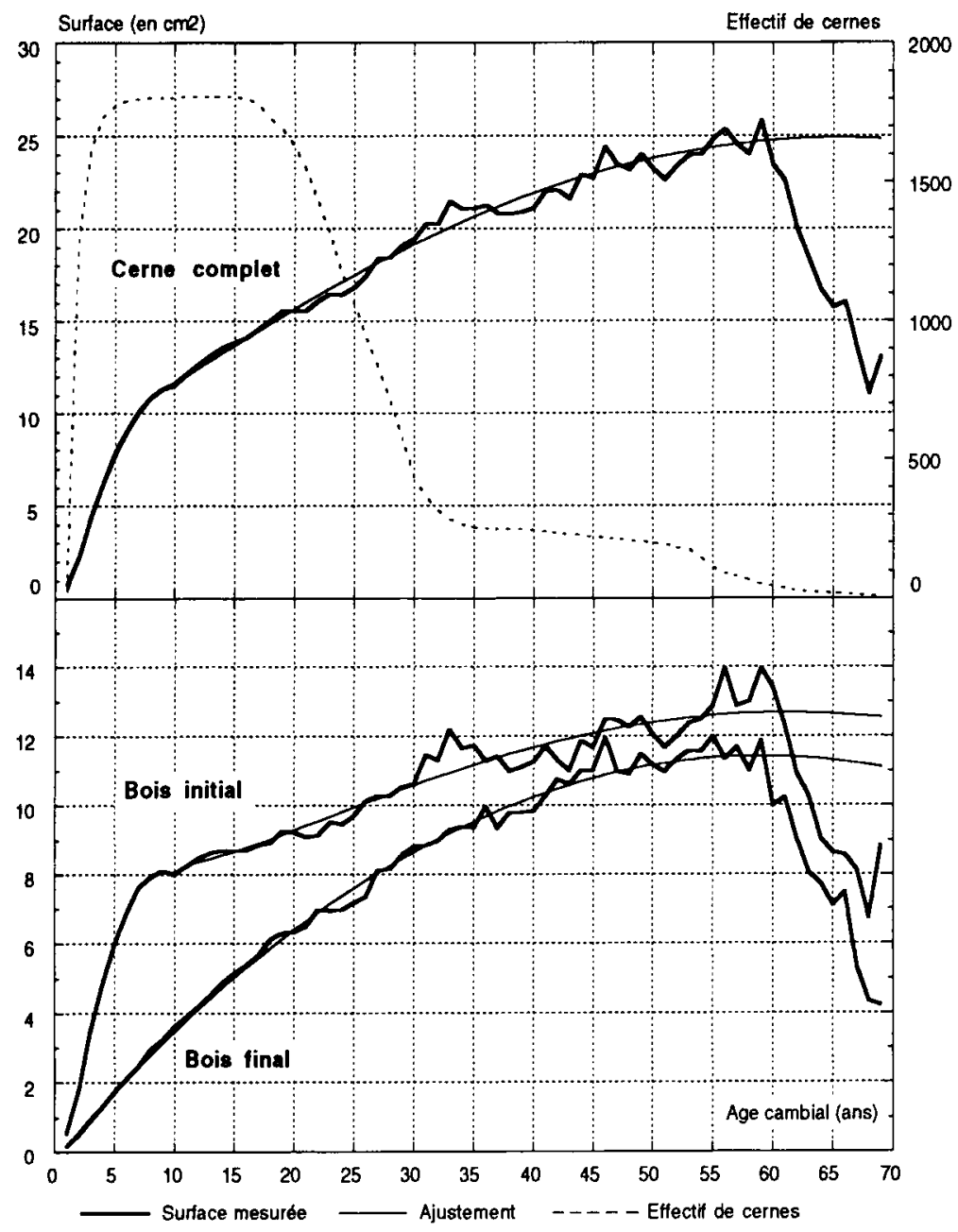

Fig 3. Accroissement moyen selon l'âge cambial, ajustement et effectifs de cernes pour le cerne complet, le bois initial et le bois final.

précédemment pour le cerne complet (fig 3). En revanche, pour le bois final, la dynamique est très différente, et se caractérise par une augmentation plus faible, mais plus soute-nue, jusqu'à l'áge de 30-40 ans.

Le calcul des pourcentages moyens de bois initial et de bois final dans l'anneau de croissance montre que les très jeunes cernes sont constitués principalement de bois initial, et ce n'est qu'à partir de 40 ans que la proportion des deux compartiments se stabilise aux alentours des $50 \%$ (fig 4). II apparaît donc que la structure anatomique du cerne chez le pin laricio de Corse est très dépendante de son âge cambial. 


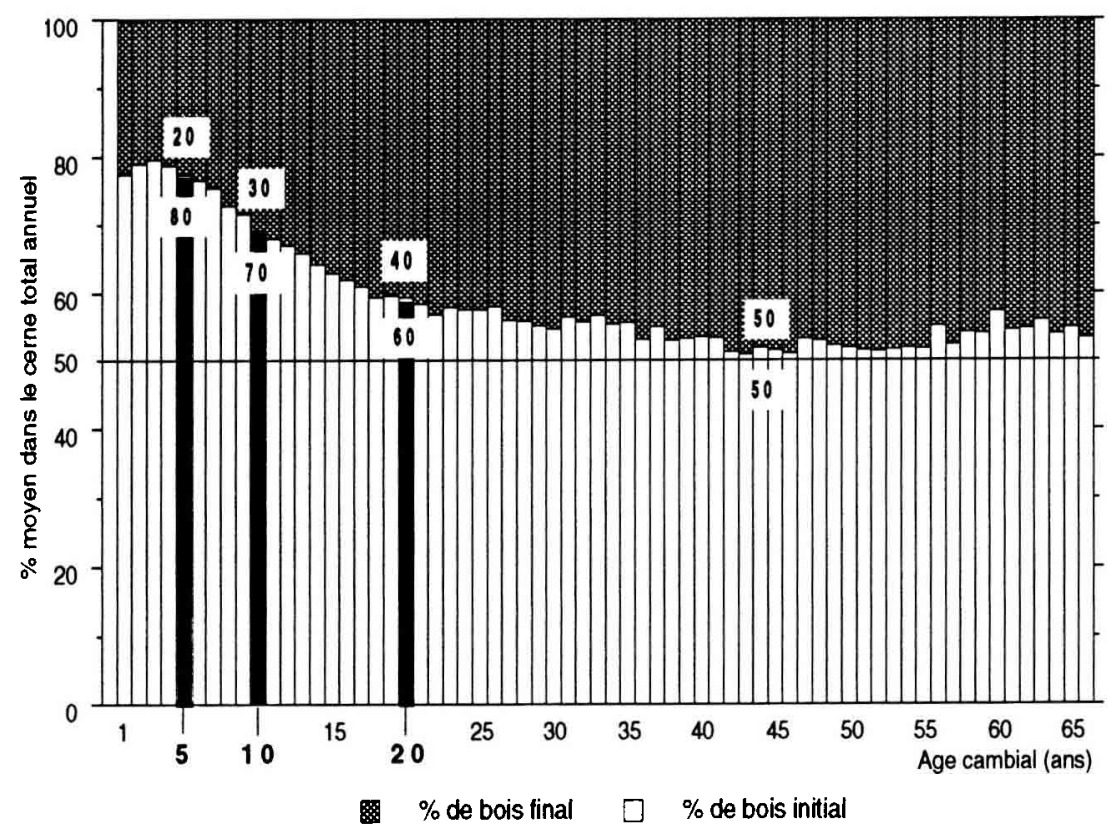

Fig 4. Pourcentage moyen (\%) de bois initial et de bois final dans le cerne total annuel selon l'âge cambial.

\section{Évolution dans le temps des indices moyens de croissance radiale}

\section{Les fluctuations annuelles et les crises de croissance}

L'importance de l'échantillon a permis de gommer la plus grande part des particularités propres à chaque individu, et les chronologies moyennes ne conservent que le signal commun à l'ensemble des arbres, c'est-à-dire essentiellement le signal climatique. Ce dernier peut être analysé année par année, ou sur une plus longue échelle de temps, en particulier dans le cas de successions d'années climatiquement défavorables. Celles-ci se traduisent par des " crises de croissance ", qui peuvent varier en durée et en intensité, au cours desquelles les arbres élaborent des cernes étroits. Quel que soit le compartiment du cerne considéré, les dépressions annuelles les plus importantes s'observent en 1939, 1952, 1962, 1969, 1976 (fig 5). Les premières crises de croissance observées se situent pendant les périodes 1951-1958 (le phénomène est particulièrement marqué en 1952 et 1956) et 1958-1965 (minimum en 1962). Celle qui a débuté en 1981 est encore plus profonde et n'est visiblement pas terminée.

La modélisation de la croissance radiale annuelle en fonction des conditions climatiques sera traitée dans un article séparé. Cependant, quelques informations sur le rôle du climat sont utiles pour faciliter l'interprétation de certains des résultats présentés dans le présent article. Ainsi, si l'on considère parallèlement la courbe des 


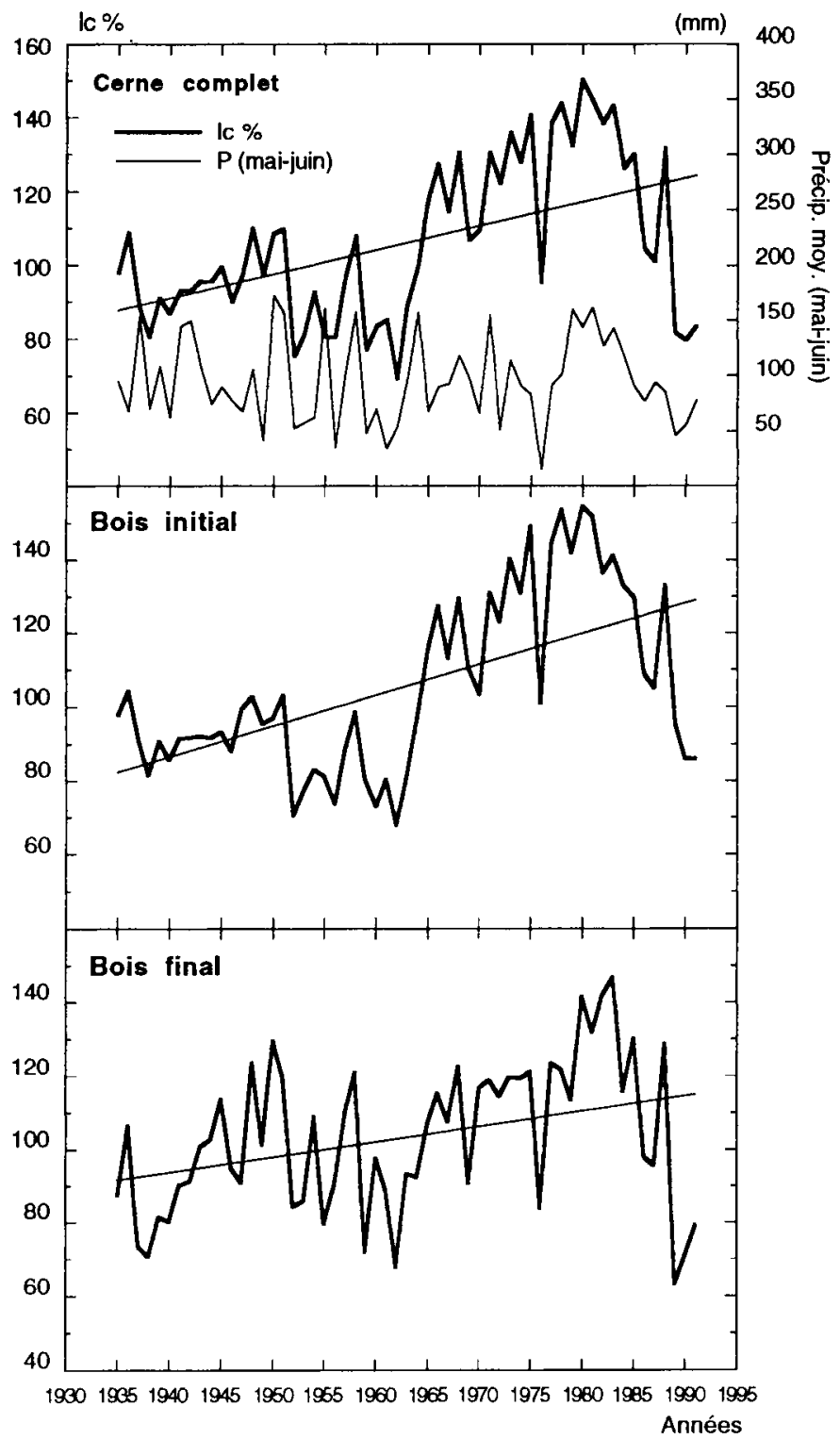

Fig 5. Évolution de l'indice moyen de croissance radiale $\left(I_{c} \%\right)$ pour le cerne complet, le bois initial et le bois final, et des précipitations moyennes de mai-juin selon la date. Les données météorologiques sont issues de la station d'Angers, et les moyennes ont été calculées sur la période 1921-1991. 
précipitations moyennes de mai-juin à Angers (station la plus représentative du climat régional, et pour laquelle on dispose des relevés les plus anciens [période 1921-1991]) et la courbe des indices moyens de croissance, on constate que les fortes crises sont associées, dans la majorité des cas, à des précipitations faibles (fig 5).

\section{Évolution à long terme de la croissance radiale}

En plus des signaux haute fréquence et moyenne fréquence s'exprimant respectivement par les variations interannuelles et les crises de croissance évoquées ci-dessus, les trois chronologies moyennes comportent un signal de basse fréquence qui se traduit par une forte tendance à long terme à l'augmentation de la croissance radiale. À partir de l'ajustement linéaire des courbes, et même en intégrant la longue période de crise qui se développe depuis le début des années 1980, l'augmentation relative depuis 1930 a été de l'ordre de $+50 \%$ si l'on considère l'évolution de la surface totale des cernes, et respectivement de +60 et $+35 \%$ si l'on considère la surface du bois initial et la surface du bois final (fig 5).

\section{Croissance radiale et aspect des houppiers}

La courbe de croissance moyenne des peuplements étudiés sur l'ensemble de l'échantillon est la résultante des comportements particuliers des arbres individuels dans les diverses placettes, qui ont toutes leurs particularités. Les préoccupations relatives à l'état sanitaire du pin laricio de Corse dans la région étudiée justifient la stratification de l'échantillon en fonction de l'aspect des houppiers et des symptômes de dépérissement observés sur le terrain. De fait, on observe une diminution importante, et ancienne, de la croissance radiale chez les arbres présentant aujourd'hui un déficit foliaire notable (fig 6). Pour les arbres de la classe 1 et de la classe 2-3, la séparation des courbes intervient dès la fin des années 1960 , ce qui coïncide avec le début d'une très longue période (10-12 ans) déficitaire au niveau pluviométrique. Pour les arbres de la classe 4-5, le niveau de croissance radiale est très inférieur à celui des autres peuplements, mais en raison de l'absence d'arbres âgés dans cette classe, la courbe moyenne ne remonte pas assez loin dans le temps pour confirmer le déterminisme essentiellement climatique du phénomène.

La différence de comportement de ces peuplements, pourtant tous soumis aux mêmes conditions climatiques, trouve en partie son explication dans certaines différences locales de disponibilité en eau, alors que la richesse minérale des sols ne semble pas intervenir. Les peuplements de classe 1 sont localisés sur les stations " humides" (indice moyen de réserve utile = 72), alors que les autres peuplements sont plutôt sur les stations " sèches " (indices moyens de réserve utile respectivement de 56 et 59 pour les classes $2-3$ et 4-5; différences significatives au seuil de $5 \%$ ).

\section{DISCUSSION ET CONCLUSION}

L'étude avait pour objectif d'analyser l'évolution à long terme de la croissance radiale moyenne du pin laricio de Corse dans la région Pays-de-la-Loire, ainsi que les relations entre état sanitaire des peuplements et croissance radiale. En toute rigueur, les courbes présentées ici ne sont valables que pour le pin laricio de Corse en région Pays-de-la-Loire. En raison des exigences propres à chaque espèce et du contexte climatique régional, il serait imprudent d'extrapoler ces résultats à une autre espèce dans la même région, ou au comportement du pin laricio dans un autre environnement climatique.

Au-delà de crises de croissance épisodiques, variables en durée et en intensité et liées à des déficits pluviométriques 


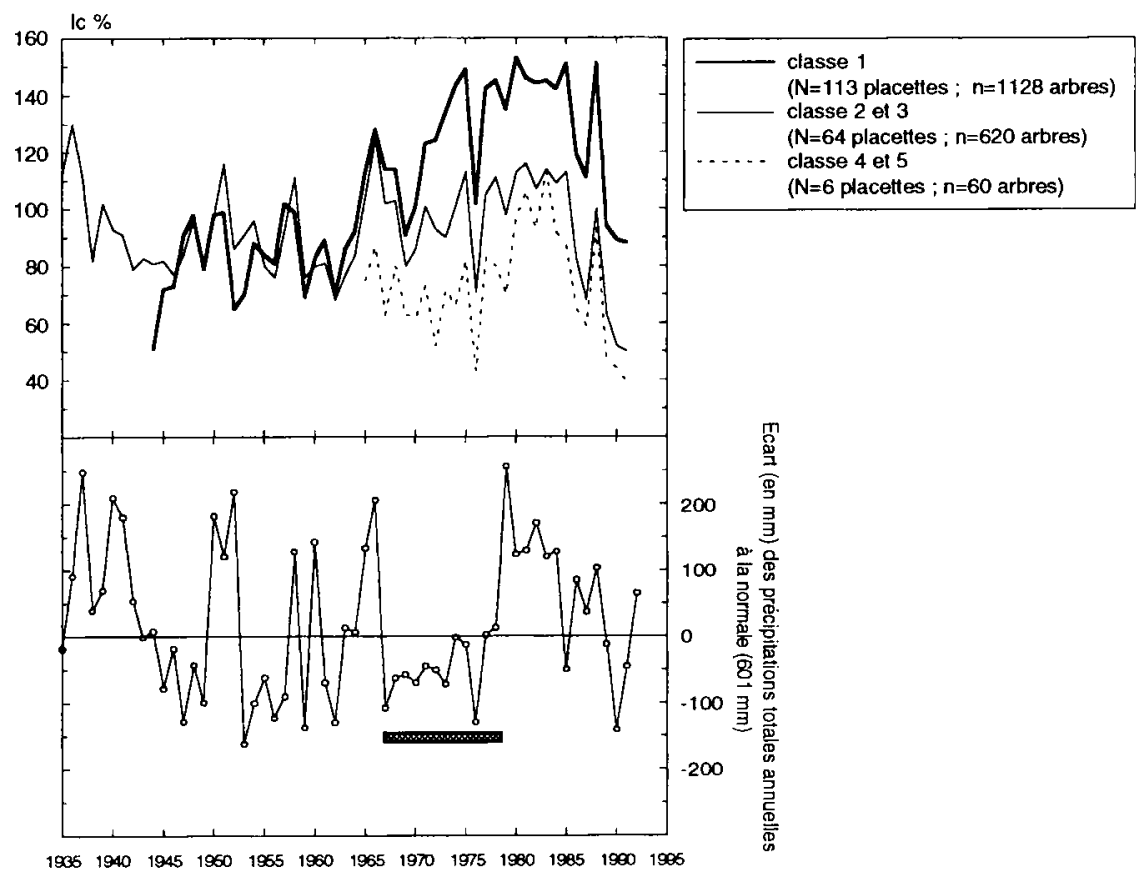

Fig 6. Évolution de l'indice moyen de croissance radiale $\left(I_{c} \%\right)$ pour les différentes classes d'état sanitaire, et de l'écart des précipitations totales annuelles à la moyenne $(601 \mathrm{~mm})$ selon la date. Les données météorologiques sont issues de la station d'Angers, et la moyenne des précipitations totales annuelles a été calculée sur la période 1921 -1991.

concomitants, le pin laricio ne présente pas sur le plus long terme de phénomène de baisse générale de la vitalité. Au contraire, la croissance radiale se caractérise en moyenne par une évolution positive importante depuis 1930. Les surfaces de cerne élaborées actuellement sont d'environ $50 \%$ plus élevées que celles des années 1930. Une telle évolution positive du potentiel de croissance a également été observée pour la même espèce en région Centre (Gilbert et Chevalier, 1994). En étudiant la croissance en hauteur dominante de cou- ples de peuplements jeunes-vieux de Sologne, Orléanais et Gâtinais, ces auteurs ont montré que les peuplements de plus de 50 ans présentent, à type de station identique, une croissance inférieure à celle des plus jeunes peuplements ; de plus, l'écart est d'autant plus important que la fertilité de la station est faible.

L'étude séparée du bois initial et du bois final met en évidence que cette tendance croissante s'exprime sur les deux compartiments du cerne annuel, avec toutefois une tendance nettement plus marquée 
pour le bois initial $(+60 \%)$ que pour le bois final $(+35 \%)$. La densité du bois initial étant plus faible que celle du bois final, la valeur de $+50 \%$ trouvée pour l'augmentation relative depuis 1930 de la surface totale des cernes doit être minorée si l'on envisage une extrapolation à la masse de bois produite. Une telle extrapolation, qui sort des objectifs de notre étude, nécessiterait la prise en compte des deux paramètres supplémentaires importants que sont la croissance en hauteur et la densité du bois. Un résultat comparable a également été obtenu par Badeau (1995) dans les futaies de hêtre des plateaux calcaires du nord-est de la France; en revanche, il n'y avait pas de différence sensible entre bois initial et bois final dans les hêtraies traitées en taillissous-futaie.

Notre étude montre que l'augmentation à long terme de la croissance radiale des peuplements forestiers ne touche donc pas seulement les forêts “ naturelles » du nord-est de la France, mais s'observe également dans un contexte climatique très différent comme celui du Centre-Ouest du pays, et dans des écosystèmes forestiers artificiels tels que celui constitué par les plantations de pin laricio de Corse. Il apparaît de plus en plus qu'il s'agit là d'un phénomène probablement très général, au moins dans les écosystèmes forestiers tempérés. À l'heure actuelle, les causes réelles en sont encore largement inconnues, et ne peuvent faire l'objet que d'hypothèses spéculatives. Si des modifications d'ordre climatique (températures et précipitations) pendant la saison de végétation sont invoquées par certains auteurs (Becker, 1989 ; Cook et al, 1991), les effets directs ou indirects sur la croissance des peuplements des dépôts atmosphériques, azotés en particulier (Kenk et Fischer, 1988 ; Bert, 1992), et de l'élévation du taux de $\mathrm{CO}_{2}$ atmosphérique (Lamarche et al, 1984) doivent être également envisagés. De plus, et particulièrement pour les espèces de reboisement, on peut également invoquer les programmes d'amélioration génétique des espèces (Roman-Amat et Arbez, 1985 ; Steinmetz, 1989), et l'amélioration des conditions de sylviculture, notamment en ce qui concerne la préparation des sols, la densité de plantation et les programmes d'éclaircies (Berben, 1986 ; Chevalier, 1990).

L'explication de la différence de comportement des deux compartiments du cerne, bois initial et bois final, est délicate et également très hypothétique. Même si aucune tendance significative à long terme n'est observable dans les données météorologiques issues de la station d'Angers, on peut penser que des températures hivernales plus clémentes, dont des modèles climatiques ont démontré l'importance pour la mise en place du cerne (Lebourgeois, 1995), en réactivant plus précocement le cambium, permettraient l'allongement de la saison de végétation et favoriseraient ainsi plus particulièrement la mise en place d'un plus grand nombre de cellules de bois initial.

La mesure séparée du bois initial et final a également permis de mettre en évidence que la structure anatomique du cerne change selon l'âge de son élaboration. Dans le jeune âge, la proportion est en moyenne de $80 \%$ de bois initial et $20 \%$ de bois final. Par la suite, la différence diminue d'environ $10 \%$ tous les 10 ans, et, à partir de 40 ans, un équilibre se crée avec $50 \%$ de bois initial et $50 \%$ de bois final. Pour expliquer cette variation de structure, on peut émettre l'hypothèse que, chez les pins jeunes, la proportion élevée de bois initial, dont le rôle essentiel est la conduction de la sève, permet de répondre à la forte demande nécessaire à un développement rapide du houppier. Quand l'arbre vieillit et que le peuplement se ferme, la vitesse de croissance se stabilise. La demande est moins importante, entraînant une réduction du tissu conducteur au profit du bois final de soutien.

La très faible fréquence de cernes manquants - fréquence dont diverses études 
ont montré qu'elle est d'autant plus élevée que les symptômes de dépérissement (pertes foliaires et jaunissement) sont accusés (Becker, 1987 ; Elling, 1987 ; Bert, 1988 ; Petersen, 1992 ; Lebourgeois et al, 1993 ; Gandolfo et Tessier, 1994) - et l'augmentation globale importante de la croissance radiale sur le long terme semblent impliquer l'absence de problème généralisé de croissance du pin laricio de Corse en région Pays-de-la-Loire. Cependant, l'analyse plus détaillée des relations entre aspect actuel des houppiers et croissance met clairement en évidence que les arbres présentant actuellement un déficit de feuillage plus ou moins marqué ont une croissance nettement plus faible, qui diverge progressivement de la tendance générale depuis une vingtaine d'années. Cette baisse de croissance apparaît être en relation directe avec une succession d'années particulièrement sèches, dont les effets ont été localement amplifiés par des conditions d'approvisionnement en eau défavorables. Ces résultats rejoignent certaines observations relatives au sapin dans les Vosges et le Jura, portant sur les divergences très anciennes de la croissance dans les peuplements actuellement dépérissants ( 30 ans dans les Vosges, 50-60 ans dans le Jura), et sur l'importance du climat et des facteurs hydriques dans l'expression de la croissance radiale et l'apparition des symptômes de dépérissement (Becker, 1987 ; Bert et Becker, 1990).

La pertinence du critère densité de feuillage pour juger du niveau réel de vitalité des arbres et de leur état sanitaire n'est cependant pas absolue. En effet, dans notre étude, une note sanitaire de 2 correspondait à la présence de deux ou trois arbres légèrement défoliés (5 à $25 \%$ de perte supposée de feuillage) parmi les dix échantillonnés. Or, pour chaque classe d'état sanitaire, les indices de croissance ont été calculés sur l'ensemble des arbres de la placette, en incluant les arbres apparemment sains. Les arbres sains étant large- ment majoritaires dans la plupart des placettes, leur croissance potentiellement bonne aurait dû masquer en grande partie la moindre croissance des arbres dépérissants. Au vu de l'écart très net observé entre les courbes moyennes de croissance radiale, on peut conclure que le potentiel de croissance des arbres apparemment en bonne santé est également affecté. La notation sanitaire sur la base de la densité de feuillage apparaît donc, pour cette espèce, particulièrement délicate et trompeuse pour juger de la vitalité réelle des arbres.

Quoi qu'il en soit, il apparaît très important de suivre ultérieurement l'évolution sanitaire des peuplements des classes 2 à 5 , et ceci d'autant plus que les très fortes sécheresses du début des années 1990 ont probablement encore altéré leur capacité de résistance. De façon plus générale, il serait hautement souhaitable d'envisager à brève échéance une nouvelle investigation dendroécologique légère sur une fraction représentative de notre échantillon pour vérifier si, avec le retour d'une pluviométrie proche de la normale à partir de 1992, la croissance radiale a globalement retrouvé un niveau comparable à ce qu'il était avant le début de la crise générale très profonde survenue depuis 1986 .

\section{REMERCIEMENTS}

Les auteurs remercient le CRPF des Pays-dela-Loire (F Barbotin et B Guimbretière), l'équipe du Cemagref de Nogent-sur-Vernisson (R Chevalier, Y Dumas, JM Gilbert et B Vallée), ainsi que les techniciens du laboratoire de phytoécologie (F Gérémia, R Schipfer) pour l'aide apportée lors de la récolte des données sur le terrain.

\section{RÉFÉRENCES}

Badeau V (1995) Étude dendroécologique du hêtre ( $F a-$ gus sylvatica L) sur les plateaux calcaires de Lorraine. Influence de la gestion sylvicole. Thèse, université Henri-Poincaré Nancy-1, $203 p+$ annexes

Badeau V, Dupouey JL, Becker M, Picard JF (1995) Long-term growth trends of Fagus sylvatica $L$ in northeastern France. A comparison between high and low density stands. Acta Oecol 16, 571-583 
Becker M (1987) Bilan de santé actuel et rétrospectif du sapin (Abies alba Mill) dans les Vosges. Étude écologique et dendrochronologique. Ann Sci For 44, 379-402

Becker $M$ (1989) The role of climate on present and past vitality of silver fir forests in the Vosges mountains of northeastern France. Can J For Res 19, 11101117

Becker M, Bert GD, Bouchon J, Picard JF, Ulrich E (1994) Tendances à long terme observées dans la croissance de divers feuillus et résineux du nord-est de la France depuis le milieu du $x^{2}{ }^{e}$ siècle. Rev For Fr 46, 335-341

Berben JC (1986) Éclaircies précoces en peuplements de pin de Corse. Bull Soc Royale For Belg 94, 167-175

Bert GD (1988) Étude dendroécologique du dépérissement du sapin (Abies alba Mill) dans le Jura. Mémoire de DEA, université Nancy-I, $62 p$ + annexes

Bert GD (1992) Influence du climat, des facteurs stationnels et de la pollution sur la croissance et l'état sanitaire du sapin pectiné (Abies alba Mill) dans le Jura. Étude phytoécologique et dendrochronologique. Thèse, université Nancy-I, $200 \mathrm{p}+$ annexes

Bert GD, Becker M (1990) Vitalité actuelle et passée du sapin (Abies alba Mill) dans le Jura. Étude dendroécologique. Ann Sci For 47, 395-412

Bonneau M (1987) Genèse et contenu du programme Deforpa. In : Les recherches en France sur le dépérissement des forêts, programme Deforpa, premier rapport, Engref-Nancy, 6-10

Briffa K R (1992) Increasing productivity of 'natural growth' conifers in Europe over the last century. International Dendrochronological Symposium 'Tree rings and Environment', Department of Quaternary Geology, Lund University, 34, 64-71

Chevalier R (1990) Pour " des sylvicultures du pin laricio en région Centre ". Rapport de BTS-CNPR Marmilhat, $38 \mathrm{p}+$ annexes

Cook ER, Bird T, Peterson M, Barbetti M, Buckley B, D'arrigo $R$, Francey $R$, Tans $P$ (1991) Climatic change in Tasmania inferred from a 1089-year tree ring chronology of Huon pine. Science 253, 1266-1268

Dupouey JL, Denis JB, Becker M (1992) A new method of standardization for examining long-term trends in tree-ring chronologies. International Dendrochronological Symposium 'Tree Rings and Environment', Department of Quaternary Geology, Lund University, $34,85-88$

Durel CE, Charon D (1994) Pin laricio. Forêt-Entreprise $96,70-72$

Elling (von) W (1987) Eine Methode zur Erfassung von Verlauf und Grad der Schädigung von Nadelbaumbeständ. Eur J For Path 17, 426-440

Fritts HC (1976) Tree-Ring and Climate. Academic Press, New York, $576 \mathrm{p}$

Gandolfo C, Tessier L (1994) Analyse dendroécologique sur des sapins (Abies alba Mill) et des épicéas ( $P$ icea abies $(L)$ Karst) de différentes classes de défoliaition dans la forêt de Vigo di Ton (Trento, Italie). Ann Sci For 51, 391-406
Gilbert JM, Chevalier R (1994) Relations milieu-production du pin laricio. Étude de la croissance en hauteur. Informations Techniques du Cemagref $n^{\circ} 96$, note 2, $8 p$

Graumlich LJ (1991) Subalpine tree growth, climate, and increasing $\mathrm{CO}_{2}$ : an assessment of recent growth trends. Ecology 72, 1-11

Graumlich LJ, Brubaker LB, Grier CC (1989) Long-term trends in forest net primary productivity: Cascade mountains, Washington. Ecology 70, 405-410

Hari $\mathrm{P}$, Arovaara $\mathrm{H}$ (1988) Detecting $\mathrm{CO}_{2}$ induced enhancement in the radial increment of trees. Evidence from northern timber line. Scandin J For Res $3,67-74$

Hari P, Arovaara H, Raunemaa T, Hautojarvi A (1984) Forest growth and the effects of energy production: a method for detecting trends in the growth potential of trees. Can J For Res 14, 437-440

Kauppi PE, Mielikainen K, Kuusela K (1992) Biomass and carbon budget of European forests, 1971 to 1990. Science 256, 70-74

Kenk G, Fischer H (1988) Evidence from nitrogen fertilisation in the forest of Germany. Environ Pollut 54, 199-218

Lamarche VC, Graybill DA, Fritts HC, Rose HR (1984) Increasing carbon dioxide: tree ring evidence for growth enhancement in natural vegetation. Science $225,1019-1021$

Lebourgeois F (1995) Étude dendroécologique et écophysiologique du pin laricio de Corse (Pinus nigra Arnold ssp laricio Poiret var corsicana) en région Pays-de-la-Loire. Thèse, université de Paris-SudOrsay, $209 \mathrm{p}+$ annexes

Lebourgeois F, Becker M, Bonneau M (1993) Influence d'une fertilisation minérale sur la croissance radiale de sapinières dépérissantes dans les Vosges. Rev For Fr 55, 639-650

Pardé J, Bouchon J (1988) Dendrométrie. $2^{\mathrm{e}}$ édition, Engref Nancy, $328 p$

Petersen A (1992) The effect of soil treatment on the growth of damaged street trees. International Dendrochronological Symposium 'Tree rings and Environment', Department of Quaternary Geology, Lund University 34, 264-268

Picard JF (1995) Évolution de la croissance radiale du hêtre (Fagus sylvatica $L$ ) dans les Vosges. Premiers résultats sur le versant lorrain. Ann Sci For 52, 11-21

Roman-Amat B, Arbez M (1985) Pin laricio de Corse et de Calabre. Quelles provenances choisir? Le point sur les expériences comparatives de l'Inra. Rev For Fr 37, 377-388

Schweingruber $\mathrm{FH}$ (1985) Abrupt changes in growth reflected in tree ring sequences as an expression of biotic and abiotic influences. Inventorying and monitoring endangered forests. Iufro Conference, $\mathrm{Zu}$ rich 291-295, Birmensdorf, Eidg. Anstalt für das forstliche versuchswesen

Schweingruber FH (1992) Event years and pointer years. International Dendrochronological Symposium 'Tree rings and Environnement', Department of Quaternary Geology, Lund University 34, 288-292 
Schweingruber FH, Eckstein D, Serre-Bachet $F$ Braker OU (1989) Identification, presentation and interpretation of event years and pointer years in dendrochronology. Informal meeting of dendrochronologists from southern and central
Europe,Lourmarin,Provence,France,4May1989, 9-38

Steinmetz G (1989) La réglementation des graines et des plants. Un label de qualité pour les sylviculteurs. Forêt-Entreprise 59, 18-22 